\title{
Irrelevant learned reward associations disrupt voluntary spatial attention
}

\author{
Mary H. MacLean ${ }^{1,2}$ • Gisella K. Diaz ${ }^{1}$ - Barry Giesbrecht ${ }^{1,2}$
}

Published online: 15 April 2016

(C) The Psychonomic Society, Inc. 2016

\begin{abstract}
Attention can be guided involuntarily by physical salience and by non-salient, previously learned reward associations that are currently task-irrelevant. Attention can be guided voluntarily by current goals and expectations. The current study examined, in two experiments, whether irrelevant reward associations could disrupt current, goal-driven, voluntary attention. In a letter-search task, attention was directed voluntarily (i.e., cued) on half the trials by a cue stimulus indicating the hemifield in which the target letter would appear with $100 \%$ accuracy. On the other half of the trials, a cue stimulus was presented, but it did not provide information about the target hemifield (i.e., uncued). On both cued and uncued trials, attention could be involuntarily captured by the presence of a task-irrelevant, and physically non-salient, color, either within the cued or the uncued hemifield. Importantly, one week prior to the letter search task, the irrelevant color had served as a target feature that was predictive of reward in a separate training task. Target identification accuracy was better on cued compared to uncued trials. However, this effect was reduced when the irrelevant, and physically non-salient, reward-associated feature was present in the uncued hemifield. This effect was not observed in a second, control experiment in which the irrelevant color was not predictive of reward during training. Our results indicate that involuntary, value-driven capture can disrupt the voluntary control of spatial attention.
\end{abstract}

Mary H. MacLean

mary.maclean@psych.ucsb.edu

1 Department of Psychological and Brain Sciences, University of California Santa Barbara, Santa Barbara, CA 93106, USA

2 Institute for Collaborative Biotechonologies, University of California Santa Barbara, Santa Barbara, CA 93106, USA
Keywords Attention: Selective - Attentional capture · Cognitive and attentional control

Over the course of Steve Yantis' career, he was consistently at the leading edge of efforts to understand the perceptual, cognitive, and neural mechanisms that mediate the control of visual attention (e.g., Greenberg, Esterman, Wilson, et al., 2010; Jonides \& Yantis, 1988; Kelley, Serences, Giesbrecht, \& Yantis, 2007; Remington, Johnston, \& Yantis, 1992; Serences, Shomstein, Leber, Golay, Egeth, \& Yantis 2005; Serences \& Yantis, 2007; Yantis \& Jonides, 1984, 1990, 1996; Yantis, Schwarzbach, Serences, et al., 2002). Much of his early work contributed to the classic dichotomy that categorizes attention as either "top-down" - that is, voluntary and goal-driven - or "bottom-up" - that is, automatic and stimulus-driven (i.e., physical salience; e.g., Bundesen, 1990; Corbetta \& Shulman, 2002; Desimone \& Duncan, 1995; Itti \& Koch, 2001). Within the past decade of his life, Steve was again at the leading edge of the field, when he and his collaborators demonstrated the effects of learned reward history on attention (Anderson, Laurent, \& Yantis, 2011). Importantly, the effects of reward history are part of a larger class of factors that affect the allocation of attention that does not fit into the classic top-down/bottom-up dichotomy.

Reward history (Anderson et al., 2011; Anderson, 2014; MacLean \& Giesbrecht 2015a, b), context (Chun \& Jiang, 1998; Eckstein, Drescher, \& Shimozaki, 2006), and selection history (e.g., Kyllingsbæk, Schneider, \& Bundesen, 2001) all drive the allocation of attention, and can do so involuntarily, despite not being physically salient. In order to accommodate this evidence, it has been proposed that attention is controlled not only by current goals but also by factors that have previously guided attention toward information that was formerly goal-relevant (Anderson, 2013, 2015; Awh, Belopolsky \& 
Theeuwes, 2012; Vuilleumier, 2015). This hypothesis implies that there are two, potentially conflicting, factors driving attention: factors related to current goals, which voluntarily drive attention; and learned factors, which involuntarily drive attention much like physical salience. The current study investigated the relationship between these two factors by manipulating whether task-relevant targets were preceded by accurate information about their location and by manipulating the location of task-irrelevant features that were previously rewarded targets in a separate session. The effect of a voluntary cue on attention was reduced when a task-irrelevant, reward-associated feature was presented in the uncued hemifield. Furthermore, the spatially dependent effect of a previously rewarded, former target feature on attention was only present when following a spatially predictive voluntary cue. The results are consistent with the notion that spatial attention is driven by the interaction between one's current explicit goals, engendered by predictive spatial cues, and one's former goals, in the form of previously reward-associated features.

\section{Value-driven capture}

Visual features, such as color (Anderson et al., 2011; Hickey, Chelazzi, \& Theeuwes, 2010; MacLean \& Giesbrecht 2015a, b), orientation (Laurent, Hall, Anderson, \& Yantis, 2015), and context (Anderson, 2014), all guide spatial attention when previously associated with a reward, even when these features are not physically unique or salient in a display. This reward association can be learned when, for example, a particular target color in a visual search task is associated with a reward (Anderson et al., 2011). It has been observed repeatedly that after such reward learning, reaction time (RT) on a subsequent visual search task can be increased when the former reward-associated color is presented with a distracter compared to when it is absent (for reviews see Anderson, 2013, 2015). Target discrimination accuracy is also affected under similar conditions, such that accuracy increases when the formerly reward-associated color is present with a target and decreases when it is present with a distracter, relative to when that color is absent (MacLean \& Giesbrecht 2015a, b). These effects are observed even though the reward-associated features are neither currently task relevant, nor physically salient, and when performance is no longer rewarded, indicating that it is learned value specifically that captures attention. This effect, referred to as value-driven capture (Anderson et al., 2011), is similar to capture by physically salient features in that it is involuntary. Yet, as learned value is goal-driven, the two are not exactly the same and it is possible that they are not modulated by topdown, voluntary control of attention in the same way.

Although capture by physically salient features can be involuntary, its effect can be modulated by voluntary attentional control. For example, physically salient distracters can fail to capture attention when presented outside the current focus of attention (Belopolsky, Zwaan, Theeuwes, \& Kramer, 2007) and, under some conditions, when presented at completely task-irrelevant locations (Johnson, McGrath, \& McNeil, 2002; Sy, Guerin, Stegman, \& Giesbrecht, 2014). There is evidence of a similar effect with value-driven capture. Recent evidence suggests that capture by task-irrelevant features previously associated with reward may also be modulated by top-down control. When a rewardassociated color appeared in a flanking task-irrelevant location, where no target was ever presented, the effect on target accuracy was reduced compared to when it appeared in a task-relevant location (MacLean \& Giesbrecht 2015a, b). However, the extent to which such effects are driven by voluntary goal-driven control over spatial attention is unclear. Instead, it is possible that the size of the attentional window is not determined voluntarily (Theeuwes, Kramer, \& Belopolsky, 2004), and that ignoring stimuli outside of task-relevant space can be a stimulus-driven process.

\section{Attentional control via voluntary cueing}

Spatial cues that predict the most likely location of a subsequent target is a standard approach for investigating the effects of voluntary, goal-driven control over spatial attention (Posner, 1980). These cues lead to the volitional orienting of attention in contrast to exogenous cues, such as an abrupt onset (Jonides \& Yantis 1988; Remington, Johnston, \& Yantis, 1992; Yantis and Jonides 1984, 1990, 1996), which orient attention involuntarily. Importantly, when target location is indicated in advance by a voluntary cue, exogenous cues fail to capture attention when presented at an uncued location (Theeuwes, 1991; Yantis \& Jonides, 1990). This indicates that involuntary capture by physically salient features is modulated by the top-down attentional control enabled by voluntary spatial cues. Due to the unique goal-based aspect, voluntary spatial cues may not have the same effect on value-driven capture as on salience-driven capture.

One recent study approached this issue using a Posner cueing paradigm, with an $80 \%$ valid arrow cue that indicated the target location prior to target appearance (i.e., a voluntary cue), and a spatially non-predictive physically salient cue, in the form of a colored box, presented simultaneously with the target (i.e., an involuntary/reflexive cue; Munneke, Hoppenbrouwers, \& Theeuwes, 2015). Although the physically salient cue was spatially non-predictive, it did indicate the magnitude of reward for that trial - no reward, a small reward, or a large reward. Both cues significantly affected attention, such that RTs were slower following invalid than valid voluntary/exogenous cues. The two effects did not interact, suggesting that value-driven capture is unaffected by top- 
down voluntary attentional control, and vice versa. However, because the reward-associated feature was physically salient and the same effects were observed in the control condition where no reward was given, it is unclear the extent to which these effects are value-driven. Furthermore, the non-predictive cue was arguably task-relevant as it indicated the reward for that trial, and it is possible that the reduced validity of the voluntary cue diminished its influence. Finally, overlearned symbols such as arrows have been shown to cause automatic orienting of attention (Ristic \& Kingstone, 2012), such that they are not a pure voluntary cue. Thus, the relationship between voluntary attentional control, induced by a voluntary cue, and involuntary value-driven capture remains unclear.

\section{Current study}

In the current study we investigated the relationship between current goal-driven factors that voluntarily drive attention and former value-driven factors that involuntarily drive attention. In order to investigate this issue our study satisfied two critical conditions. First, there must be evidence of reliable valuedriven capture. This is demonstrated by decreased performance on trials where a reward-associated feature, learned in a preceding training task, was present as compared to performance on trials where it was absent in a visual search task. Second, there must be evidence that the voluntary cues engaged attention in the same visual search task. This is demonstrated by improved performance on cued trials as compared to performance on uncued trials.

To ensure that value-driven capture was completely taskirrelevant, we used a reward-training task that preceded the visual search task. We also separated the reward training and visual search tasks by 1 week, to further diminish the relevance of reward in the visual search task. Importantly, the reward-associated features were physically non-salient, thus ruling out the contribution of stimulus-driven capture effects. An additional control experiment was conducted during which no rewards were provided during training, to assess the contributions of selection history (Anderson et al., 2011).

To maximize the voluntary nature of top-down control via cueing in our paradigm, we used arbitrarily chosen letters and symbols $(\mathrm{x}, \mathrm{v}$, and $=)$ to indicate target location. These symbols, in contrast to arrows, do not have an overlearned spatial component and thus require intentional, voluntary interpretation to guide attention to a particular location. Further to this point we made these cues $100 \%$ valid, and informed participants of their perfect validity. Two cues $(\mathrm{x}, \mathrm{v})$ indicated the hemifield (left or right), in which the target would appear; one $(=)$ provided no spatial information. This permitted us to examine the effects of reward-driven capture within the cued hemifield, where attention would voluntarily be directed, and outside the cued hemifield.

\section{Methods: Experiment 1}

\section{Participants}

Forty-six undergraduate students (mean age $=21$ years; 31 females) volunteered to participate in this study. All were recruited from the Psychological and Brain Sciences research participation pool at the University of California, Santa Barbara (UCSB). Participants received research participation credit $(n=18)$ or were paid $\$ 10 / \mathrm{h}(n=28)$. All procedures were approved by the UCSB Human Subjects Committee.

\section{Apparatus}

Stimuli were generated using the Psychophysics Toolbox extensions (Brainard, 1997; Kleiner et al., 2007; Pelli, 1997) on a Mac Mini (Apple, Inc.) running OSX (10.8.5) and MATLAB R2013a (Mathworks). Stimuli were presented on a color CRT monitor $(36 \times$ $27 \mathrm{~cm}$ ), set to a resolution of $1,280 \times 1,024$ and $a$ refresh rate of $60 \mathrm{~Hz}$. The viewing distance was 110 $\mathrm{cm}$.

\section{Procedure}

Participants completed the study in two separate sessions, exactly 1 week apart. In the first session, participants completed a training task where they learned to associate specific colors (red and blue) with a high $(\$ 0.05)$ or low $(\$ 0.01)$ monetary reward. In the second session, participants completed a cueing task where we measured the performance benefit of using central spatial cues in the presence and absence of a previously rewarded color. Importantly, the previously rewarded colors were no longer rewarded or relevant in the cueing task. In the first session, participants also completed a change detection task for a measure of visual working memory capacity, a BIS/ BAS questionnaire (Carver \& White, 1994) for a measure of reward sensitivity, and a demographic questionnaire.

\section{Training task}

Each trial began with a white (RGB: 255, 255, 255) centered fixation cross $\left(0.21^{\circ}\right.$ of visual angle width/height $)$ that was presented for a randomly determined duration of $400 \mathrm{~ms}$, $500 \mathrm{~ms}$, or $600 \mathrm{~ms}$. The stimulus display followed and was presented for $600 \mathrm{~ms}$ (see Fig. 1a). Each stimulus display contained six differently colored rings $\left(0.63^{\circ}\right.$ diameter $)$ presented in a circular arrangement equidistant from the fixation cross (radius $=1.09^{\circ}$ from the center of the screen to the center of each ring) and each other ( $1.09^{\circ}$ center-to-center $)$. The possible colors of the rings were red $(206,0,0)$, blue $(0,0,250)$, orange $(197,98,0)$, teal $(0,118,118)$, brown $(169,85,0)$, green $(0,141,0)$, gold $(118,118,0)$, violet $(128,0,192)$, and 
(a)

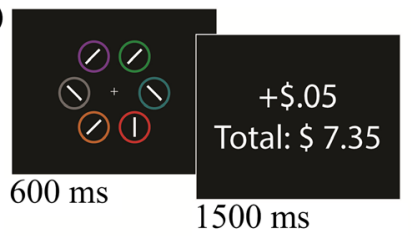

(b)

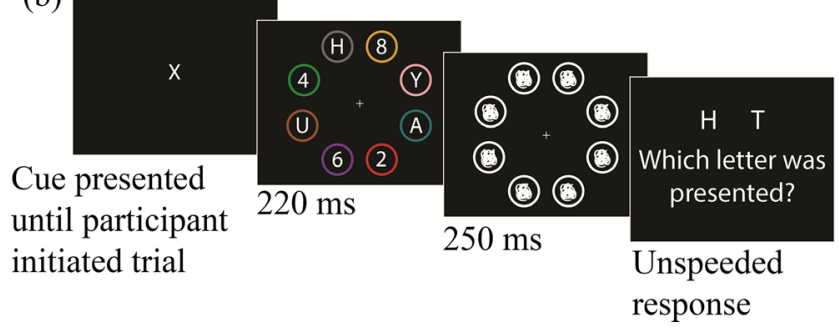

Fig. 1 (a) Example trial from the Reward Training task. Participants were instructed to look for the red or blue target circle (red in example), and make a speeded response to indicate whether the line segment in the target circle was oriented vertically (as in the example), or horizontally. One target color was associated with a larger reward of $\$ 0.05$ (as in the example), and the other with a smaller reward of $\$ 0.01$. (b) Example trial from the Cueing task. Participants were first presented with either a predictive cue (as in the example) indicating in which hemifield the target would appear (100\% valid), or an uninformative cue ("="). Participants self-initiated the presentation of the stimulus display, which was presented briefly and followed by a pattern mask. Participants then made an unspeeded response to indicate which of two letters had been presented in the preceding display. On half the trials one of the distracter numbers was presented inside a ring in a former target/reward-associated color (red, as in the example, or blue)

gray $(110,110,110)$. Each stimulus display contained five distracter rings and one target ring. The target ring was red on half of the trials and blue on the other half of the trials. The color of each distracter ring was drawn randomly without replacement on each trial from the remaining colors. The location of the target ring was determined randomly on each trial from the six possible locations. Each colored ring contained a white line segment $\left(0.47^{\circ}\right.$ long, $0.05^{\circ}$ thick). The line segment inside the target ring was vertical on half of the trials and horizontal on the other half of the trials. For the distracter rings, the line segment was either angled $45^{\circ}$ to the left or $45^{\circ}$ to the right. Participants were instructed to indicate the orientation of the line segment inside the target ring by pressing the letter " $\mathrm{z}$ " on a keyboard if it was vertical or the letter " $\mathrm{m}$ " if it was horizontal. Participants were also instructed to answer as quickly and accurately as possible. Each response was followed by a feedback display that was presented for 1 , $500 \mathrm{~ms}$. The feedback display stated the reward outcome of the previous trial and the amount of money earned thus far. On high-reward trials, participants could win $\$ 0.05$ if they answered correctly, and $80 \%$ of the high-reward trials had the potential to be rewarded. On low-reward trials, participants could win $\$ 0.01$ if they answered correctly, and $20 \%$ of the low-reward trials had the potential to be rewarded. The target color (i.e., red or blue) associated with the high reward was counterbalanced across participants. Stimuli were presented on a black $(0,0,0)$ background. Participants were not informed about the reward contingencies, but they were informed that they had the chance to win money on each trial. The training task began with a practice block of 20 trials that were not rewarded, followed by ten blocks of 80 trials each for a total of 800 trials. Each block contained 40 high-reward and 40 low-reward trials that were mixed randomly within each block. Trials were categorized into high- and low-reward trials, and mean accuracy and mean RTs were calculated for each type of trial. Learning in the training task was defined as higher accuracy and faster RTs in the high-reward trials compared to the low-reward trials.

\section{Cueing task}

Each trial began with a white cue letter or symbol $\left(0.26^{\circ}\right.$ width, $0.31^{\circ}$ height) that was presented at fixation until the participants pressed the spacebar to show the stimulus display (see Fig. 1b). The cues were the letters "X," "V," and the symbol " $=$ ". Each cue contained pre-specified information about the upcoming trial. For half of the participants, the letter "X" cued the left hemifield of the stimulus display, indicating that the target would appear in the left hemifield. Similarly, the letter "V" cued the right hemifield of the stimulus display, indicating that the target would appear in the right hemifield. For the other half of the participants, the mapping was reversed ("X" = right; "V" = left). The cues were $100 \%$ valid. For all participants, the symbol "=" did not cue either side of the display and the target could appear on either side with equal probability. After participants initiated the trial, a white fixation cross was presented for $500 \mathrm{~ms}$. The stimulus display was then presented for $220 \mathrm{~ms}$. Each stimulus display contained eight differently colored rings $\left(0.63^{\circ}\right.$ diameter $)$ presented in a circular arrangement equidistant from the fixation cross (radius $=1.30^{\circ}$ from the center of the screen to the center of each ring) and each other ( $1.09^{\circ}$ center-to-center). The colors of the rings were red $(206,0,0)$, blue $(0,0,250)$, orange $(245,145,33)$, teal $(0,118,118)$, brown $(160,82,45)$, green $(0,141,0)$, gold $(218,165,32)$, violet $(128,0,192)$, gray $(110$, $110,110)$, and pink $(238,162,173)$. Half of the colored rings contained a white letter $\left(0.26^{\circ}\right.$ width, $0.31^{\circ}$ height $)$ drawn randomly without replacement from among the following: A, E, F, H, J, K, L, M, N, P, R, T, U, Y, while the other half contained a white number $\left(0.26^{\circ}\right.$ width, $0.31^{\circ}$ height $)$ drawn randomly without replacement from the numbers 1-9. Participants were instructed to maintain fixation and pay attention to the letters, while ignoring the numbers and colored rings. The stimulus display was followed by white pattern masks, presented for $250 \mathrm{~ms}$. The pattern masks were created using the symbols \#,\%, @, and \& presented on top of each other to replace the letters and numbers. The colored rings were replaced with white rings. The response display was then 
presented until a response was given. The response display contained two letters, only one of which had been presented (the target). The second letter was chosen randomly from among the letters that had not been presented. Participants were asked to indicate which of the two letters had been presented by pressing that letter on the keyboard. Trials were separated by a $500-\mathrm{ms}$ display containing only a fixation cross. Stimuli were presented on a black background.

\section{Design}

The cueing task began with a practice block of ten trials, followed by eight blocks of 128 trials each for a total of 1 , 024 trials. On half of the trials, one of the distracter rings containing a number was rendered in a previously rewarded color (i.e., red or blue). On the other half of the trials all the colored rings were rendered in colors that were not rewarded in the training task. Additionally, half of all trials were cued, such that they contained either the " $\mathrm{X}$ " or " $V$ " cue, which was predictive of the target hemifield. The other half of the trials were uncued, containing the "=" cue and giving no information about the target location. The factors of distracter type (rewarded or not) and cue type (cued, i.e., predictive, or uncued), and target location (left or right hemisphere) were fully crossed.

Trial types were distributed evenly between the blocks and mixed randomly within each block. Participants were informed about the cues and their validity before beginning the task. Mean accuracy was calculated for the different conditions as proportion correct. The presence of a cueing effect was measured by comparing accuracy between cued and uncued trials, while the presence of a capture effect was measured by comparing performance between trials that contained a previously rewarded color and trials that did not contain any previously rewarded colors.

\section{Participant attrition}

In Experiment 1, a total of 46 participants completed the first session, but only 34 participants returned for the second session. Our analyses include only participants that completed both sessions. Additional participants were removed from our analyses due to performance at or below chance level in the training task $(n=11)$ or cueing task $(n=3)$, for a final sample of 20 participants.

\section{Methods: Experiment 2}

\section{Participants}

Forty-one different undergraduate students (mean age $=20$ years; 23 females) volunteered to participate in this study.
All were recruited from the Psychological and Brain Sciences research participation pool at UCSB. All participants received research participation credit. All procedures were approved by the UCSB Human Subjects Committee.

\section{Training task}

The training task was the same training task from Experiment 1 , but there was no potential for reward. Instead of the reward feedback screen from Experiment 1, a blank screen was presented for $1,500 \mathrm{~ms}$.

\section{Cueing task}

The cueing task was the same cueing task from Experiment 1.

\section{Participant attrition}

In Experiment 2, a total of 41 participants completed the first session, but only 39 participants returned for the second session. Our analyses include only participants that completed both sessions. Additional participants were removed from our analyses due to performance at or below chance level in the training task $(n=18)$ or cueing task $(n=1)$, for a final sample of 20 participants.

\section{Experiment 1: Results}

\section{Reward training}

Hypotheses In order to demonstrate that the rewardassociation was learned, performance on the Reward Training task, either RT or target accuracy (proportion correct), must differ according to the reward contingencies. Specifically, if the reward-association was learned then RTs should be faster and/or accuracy higher to the target associated with the higher magnitude of reward than the target associated with the lower magnitude of reward.

Reaction time A paired $t$ test was used to examine RTs on correct trials of the Reward training task between low- and high-reward conditions. RTs were significantly faster to targets associated with a high reward $(M=424 \mathrm{~ms}, S E M=12$ $\mathrm{ms})$ than to targets associated with a low reward $(M=435 \mathrm{~ms}$, $\left.S E M=12 ; t(19)=3.86, p=.001, \eta^{2}=.44\right)$.

Accuracy A paired $t$ test was used to examine the proportion of correct trials on the Reward training task between low- and high-reward conditions. The proportion correct was significantly higher for targets associated with a high reward $(M=.69, S E M=.02)$ than for targets associated 
with a low reward $(M=.65, S E M=.02 ; t(19)=3.88, p=.001$, $\left.\eta^{2}=.44\right)^{1}$

\section{Cued visual search task}

Hypotheses In order to test our hypotheses we performed three separate analyses. The purpose of the first analysis was to determine (1) whether there was a significant value-capture effect and (2) whether there was a significant voluntary cueing effect. This analysis consisted of a repeated-measures analysis of variance (ANOVA) to examine proportion correct on the Cued Visual Search Task as a function of learned value of distracter feature (none, low, or high), and cue condition (uncued or cued).

The purpose of the second analysis was to determine whether the voluntary cueing and involuntary value-capture effects interact. Specifically, whether the spatial congruence of the reward-associated distracter with the cued hemifield (congruent $=$ same cued hemifield, incongruent $=$ opposite cued hemifield) affected the performance benefit derived from the voluntary spatial cue. This is similar to the approach used by Munneke et al. (2015), who examined performance as a function of whether the voluntary cue and reward-associated distracters validly or invalidly cued the target location. When their voluntary cue was valid and the rewardassociated distracter was invalid, this would be equivalent to our incongruent condition. When their voluntary cue was valid and the reward-associated distracter was also valid, this would be equivalent to our congruent condition. This analysis consisted of a repeated-measures ANOVA to examine proportion correct on the Cued Visual Search Task as a function of spatial congruence of the reward-associated feature with the cued hemifield (congruent, incongruent, or absent), and cue condition (uncued or cued). A significant interaction would indicate that involuntary value-driven capture is modulated by the voluntary allocation of attention following a spatial cue.

Finally, in the last analysis we investigated the role of spatial offset in the effects of value-driven capture and voluntary attention. It is possible that the spatial distance of the reward-associated distracter from the target may modulate both effects, and perhaps even their interaction. For this analysis we performed a repeatedmeasures ANOVA to examine proportion correct on the Cued Visual Search Task as a function of spatial offset

\footnotetext{
${ }^{1}$ The overall effect of reward condition on accuracy in the Reward Training task was modulated by the type of compensation that participants received $\left(F(1,18)=4.93, p=.039, \eta^{2}=.22\right)$, such that it was only significant for those participants who were paid for their participation $(n=$ $12, p<.001)$, and not for those who received course credit $(n=8, p=$ .340). This was not the case for the effect of reward condition on reaction time, and, importantly, there were no significant main effects or interactions with compensation type in the Cued Visual Search Task (all $p$ 's $>$ .1).
}

of the reward-associated distracter from the target $\left(45^{\circ}\right.$, $90^{\circ}$, or $135^{\circ}$ ), congruence (congruent or incongruent), and cue condition (cued or uncued). Note that the absent condition was excluded from this analysis, as it does not contain the factor of offset.

Value-driven capture There was a significant main effect of learned value $\left(F(2,38)=6.76, p=.003, \eta^{2}=.26\right.$; see Fig. 2$)$, such that accuracy was significantly reduced when either a high $(M \Delta=.035, S E M \Delta=.009, p=.012)$ or low $(M \Delta=.021$, $S E M \Delta=.008, p=.013)$ reward-associated distracter was present in the display as compared to when no reward-associated distracter was present. However, while accuracy was different, it did not differ significantly between high- and low-rewardassociated conditions $(M \Delta=.014$, SEM $=.012, p>.250)$. Thus, there was a reliable value-driven capture effect, but the magnitude of the reward did not reliably modulate the effect.

Voluntary cueing There was a significant main effect of cue condition $\left(F(1,19)=44.89, p<.001, \eta^{2}=.70\right.$; see Fig. 2$)$, such that accuracy was significantly higher on cued trials than on uncued trials $(M \Delta=.095$, SEM $\Delta=.014)$. Thus, there was a reliable voluntary cueing effect.

There was no significant interaction between learned value and cue condition in this analysis $(F(2,38)=$ $\left.1.95, p=.156, \eta^{2}=.09\right)$. However, this analysis does not consider the factor of spatial congruence, and thus the lack of interaction indicates that overall, regardless of spatial congruence, the two effects are independent.

Spatial congruence There was a significant main effect of spatial congruence $\left(F(2,38)=10.13, p<.001, \eta^{2}=.35\right.$; see Fig. 3), such that accuracy was significantly reduced when the spatial location of the reward-associated distracter was incongruent with the target hemifield as compared to when it was absent $(M \Delta=.041, S E M \Delta=.008, p<.001)$, and compared to when it was congruent with the target hemifield $(M \Delta=.026$, SEM $\Delta=.011, p=.031)$. However, accuracy did not differ significantly when the spatial location of the rewardassociated distracter was congruent with the target hemifield as compared to when it was absent $(M \Delta=.015, \operatorname{SEM} \Delta=$ $.008, p=.072)$. This indicates that value-driven capture was only reliable when the target and the reward-associated distracter were in opposite hemifields.

This result, however, does not itself indicate that the voluntary allocation of attention via a spatial cue modulated the value-driven capture effect. The spatial cue was $100 \%$ valid, thus the hemifield of the target and the cued hemifield are confounded, because the target will always appear in the cued hemifield on cued trials. As such, it is possible that it is congruence with the target hemifield and not the cued hemifield that drives the observed effects. However, because we have cued and uncued conditions, we can examine whether the 
Fig. 2 Value/selection-driven capture results from Experiments 1 (reward) and 2 (control). Error bars are SEM
Experiment 1: Reward

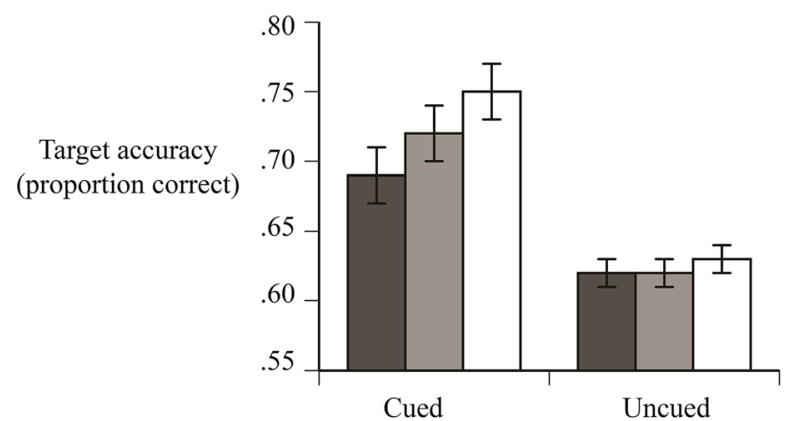

Experiment 2: Control

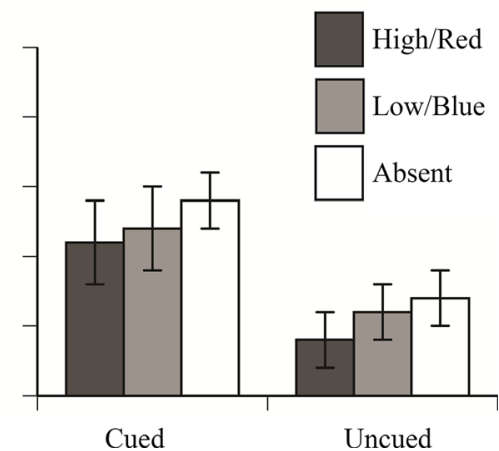

spatial congruence effect was modulated by the cueing condition (uncued vs. cued). If so, this would indicate that it was congruence with the cued hemifield and not the target hemifield that drives the effects. Critically, there was a significant interaction between spatial congruence and cue condition $\left(F(2,38)=6.15, p=.005, \eta^{2}=.24\right)$, such that the spatial congruence effect was significant $(F(2,38)=16.32, p<.001$, $\left.\eta^{2}=.65\right)$ in the cued condition, but not in the uncued condition $\left(F(2,38)=1.07, p>.250, \eta^{2}=.05\right)$. This indicates that the value-driven capture/spatial congruence effect was only reliable when attention was voluntarily oriented to the target hemifield. When attention could not be focused on the cued hemifield before the target appeared, the reward-associated distracter failed to capture attention. From another perspective, the voluntary cueing effect, although significant in all conditions (all $p$ 's $<.01$ ), was significantly reduced in the incongruent condition as compared to the congruent $(M \Delta=$ $.056, S E M \Delta=.02, p=.012)$ and absent conditions $(M \Delta=$ $.051, S E M \Delta=.015, p=.008)$, while it did not differ between the congruent and absent conditions $(M \Delta=.005, S E M \Delta=$ $.015, p>.250)$. Thus, the voluntary allocation of attention following a spatial cue was only disrupted by value-driven capture when the reward-associated distracter appeared outside of the cued hemifield.

Spatial offset There was no main effect of spatial offset $(p>.250)$; however, there was a significant interaction of spatial congruence and spatial offset $(F(2,38)=10.81, p<.001$, $\eta^{2}=.36$; see Fig. 4). This interaction was such that performance decreased with increasing spatial offset $\left(45^{\circ}>90^{\circ}>\right.$ $\left.135^{\circ}\right)$ when the reward-associated distracter appeared in the cued hemifield, but increased $\left(45^{\circ}<90^{\circ}<135^{\circ}\right)$ when it appeared in the uncued hemifield. The higher order interaction with cue condition did not reach traditional levels of significance $(p=.161)$. However, in a post-hoc analysis using two repeated-measures ANOVAs, the interaction of spatial offset and spatial congruence was significant in the cued condition $\left(F(2,38)=10.72, p<.001, \eta^{2}=.36\right)$, but not in the uncued condition $(p=.246)$. While the presence of the interaction in the cued condition and not in the uncued condition must be interpreted with caution given the lack of the higher order interaction, the result is consistent with the conclusion that it is the congruence with the cued hemifield, and not the target hemifield, which modulates the effect of spatial offset.

\section{Experiment 2: Control results}

\section{Control training}

Hypotheses Unlike in Experiment 1, we would not expect to see any differences in performance, either in RT or accuracy,
Fig. 3 Spatial congruence effects from Experiments 1 (reward) and 2 (control). Error bars are SEM
Experiment 1: Reward

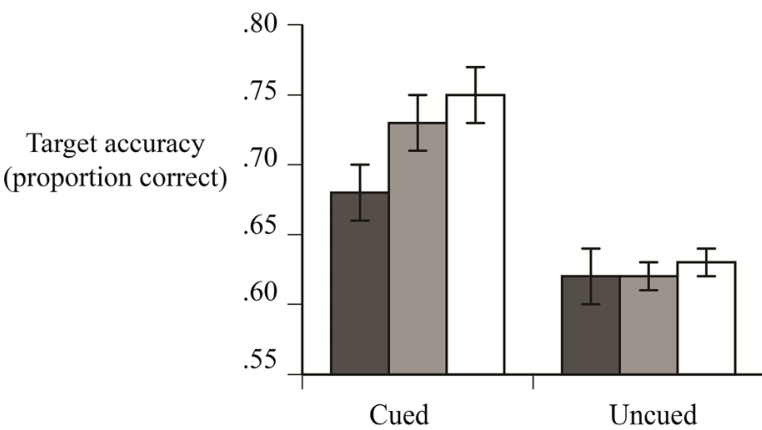

Experiment 2: Control

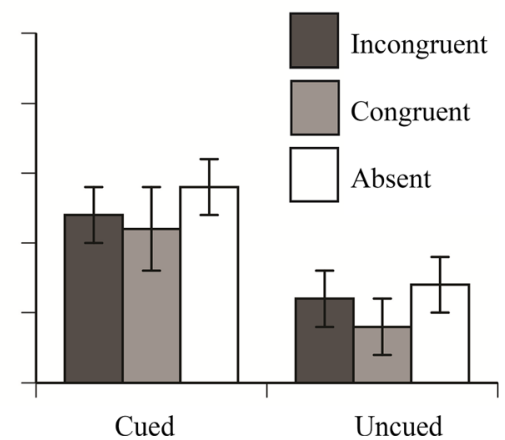


Fig. 4 Spatial offset effects from Experiments 1 (reward) and 2 (control). Errors bars are SEM
Experiment 1: Reward

Experiment 2: Control

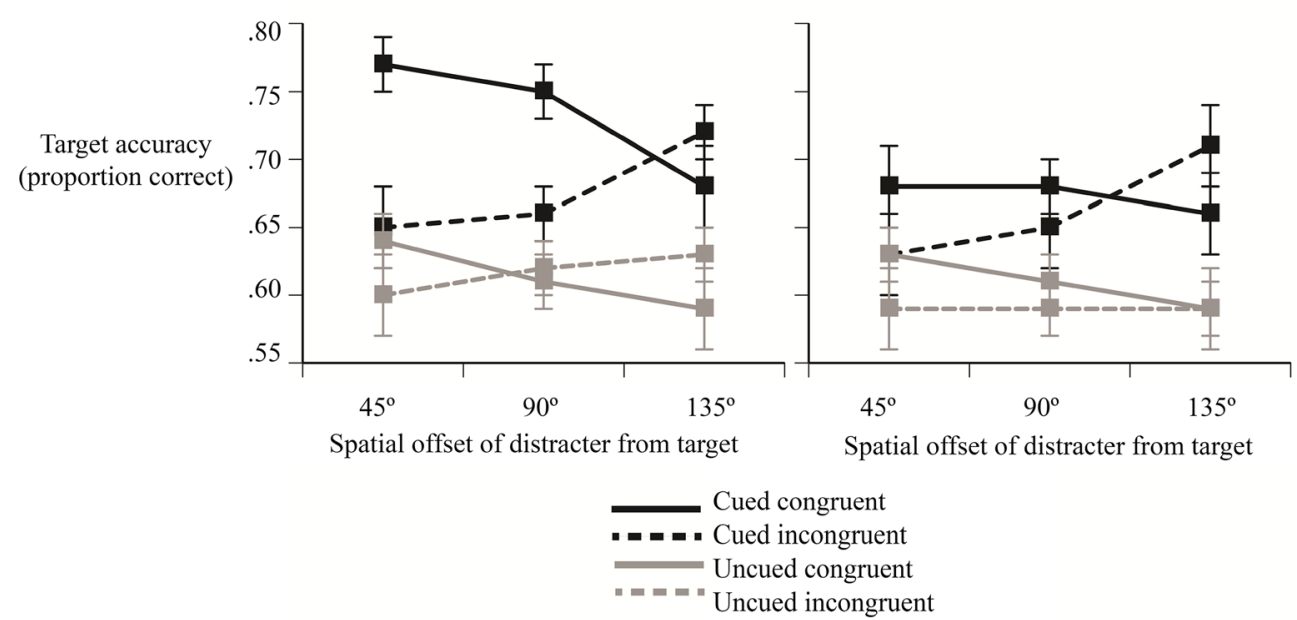

between the two target conditions (red and blue), as there were no reward contingencies to be learned in the control experiment version of the task. Any differences would only be due to the physical differences between the target conditions, i.e., that one target color was more physically salient than the other.

Reaction time A paired $t$ test was used to examine RTs on correct trials of the Control Training task between red and blue target conditions. RTs were not significantly different to red targets $(M=492 \mathrm{~ms}, S E M=6 \mathrm{~ms})$ than to blue targets $\left(M=491 \mathrm{~ms}, S E M=6 ; t(19)=.95, p>.250, \eta^{2}=.05\right)$.

Accuracy A paired $t$ test was used to examine the proportion of correct trials on the Control Training task between red and blue target conditions. Proportion correct was not significantly different for blue targets $(M=.67, S E M=.02)$ compared to red targets $\left(M=.65, S E M=.02 ; t(19)=2.06, p=.053, \eta^{2}=.18\right)$.

\section{Cued visual search task}

Hypotheses If the effects observed in Experiment 1 are due to selection history rather than reward history, i.e., that the reward-associated colors were also formerly targets/task relevant, then we should observe the same pattern of results in Experiment 2 as in Experiment 1. On the other hand, if the effects of Experiment 1 are due to either reward history or the combination of reward and selection histories, then the effects of Experiment 2 should differ from those of Experiment 1. Thus, any effects unique to Experiment 1 can be ascribed to the effects of reward history and/or the combination of reward and selection histories. Note that this control experiment does not control for the possibility that the effects observed in Experiment 1 are due to motivational salience effects generated by the receipt of rewards. However, Sali, Anderson, and Yantis (2014) demonstrated in multiple experiments using a similar paradigm that motivational salience does not account for value-driven capture.

In order to test these hypotheses, we performed the same analyses as in Experiment 1, the only difference being that instead of high- and low-reward conditions we have red and blue conditions.

Selection-driven capture There was a significant main effect of distracter type $\left(F(2,38)=4.07, p=.025, \eta^{2}=.18\right.$; see Fig. 2), such that accuracy was significantly reduced when either a red $(M \Delta=.026, S E M \Delta=.008, p=.006)$ or blue $(M \Delta=.018, S E M \Delta=.007, p=.015)$ distracter was present in the display as compared to when no distracter was present. Accuracy did not differ significantly between red and blue distracter conditions $(M \Delta=.008, S E M \Delta=.012, p>.250)$. Thus, there was a reliable selection-driven capture effect. This control analysis indicates that selection history is sufficient to have caused the effects attributed to value-driven capture in Experiment 1. However, it remains unclear whether selectiondriven effects are similarly affected by a voluntary spatial cue, as when the distracter is also reward-associated as in Experiment 1.

Voluntary cueing As in Experiment 1, there was a significant main effect of cue condition $(F(1,19)=22.66$, $p<.001, \eta^{2}=.54$; see Fig. 2$)$, such that accuracy was significantly higher on cued trials than on uncued trials $(M \Delta=.066, S E M \Delta=.014)$. Thus, there was a reliable voluntary cueing effect. As in Experiment 1, there was no significant interaction of distracter type and cue condition in this analysis $(p=.994)$.

Spatial congruence There was a significant main effect of spatial congruence $\left(F(2,38)=6.40, p=.004, \eta^{2}=.25\right.$; see Fig. 3), such that accuracy was reduced when the spatial location of the distracter was incongruent with the target 
location as compared to when it was absent $(M \Delta=.030$, $S E M \Delta=.005, p<.001)$. Unlike in Experiment 1 , accuracy did not differ significantly between congruent and incongruent conditions $(M \Delta=.015, S E M \Delta=.01, p=.072)$. However, as in Experiment 1, accuracy did not differ significantly between congruent and absent conditions either $(M \Delta=.015$, $S E M \Delta=.008, p=.088)$. This indicates that selection-driven capture, like reward-driven capture in Experiment 1, was only reliable when the target and the reward-associated distracter were in opposite hemifields.

There was no significant interaction of spatial congruence and cue condition $(p>.250)$. This indicates that the selectiondriven capture/spatial congruence effect was unaffected by the voluntary allocation of attention via a spatial cue. Similarly, unlike in Experiment 1, the voluntary allocation of attention following a spatial cue was unaffected by selection-driven capture. These results indicate that while involuntary reward-driven capture and voluntary attention interact, selection-driven capture and voluntary attention do not.

Spatial offset As in Experiment 1, there was no main effect of spatial offset $(p=.09)$. However, unlike in Experiment 1 , the interaction of spatial congruence and spatial offset was not significant ( $p=.146$; see Fig. 4 ), nor was the higher order interaction with cue condition $(p=.303)$. Thus, spatial offset did not reliably modulate any of the effects of selection-driven capture, unlike value-driven capture.

\section{Cross-Experiment Analysis}

In order to further investigate the difference in the effects observed between Experiments 1 and 2, we repeated the analyses performed for Experiments 1 and 2 with the additional between-subjects factor of experiment (1/reward or 2/control). Any significant interactions with experiment will indicate that the effect is significantly modulated by the presence of a reward-association.

\section{Reward/selection-driven capture}

For the purpose of this experiment we randomly assigned the participants in Experiment 2 (control) to one of two groups. In one group the red distracter condition was treated as the highdistracter condition, and the blue distracter condition was treated as the low-distracter condition. These assignments were reversed in the other group. There was a significant main effect of distracter type $\left(F(2,76)=10.65, p<.001, \eta^{2}=.22\right)$, such that accuracy was significantly reduced when either a high/red/blue $(M \Delta=.031, S E M \Delta=.006, p<.001)$ or low/ $\mathrm{red} / \mathrm{blue}(M \Delta=.02$, SEM $\Delta=.005, p=.001)$ distracter was present in the display as compared to when no distracter was present. Accuracy did not differ significantly between distracter conditions $(M \Delta=.011, S E M \Delta=.009, p=.204)$.
The interaction of distracter condition with experiment was not significant $(p>.250)$. Thus, as expected given the results of the two Experiments, there was a reliable capture effect overall, which is not modulated by the presence of a rewardassociation.

\section{Voluntary cueing}

There was a significant main effect of cue condition $(F(1$, $\left.38)=65.34, p<.001, \eta^{2}=.63\right)$, such that accuracy was significantly higher on cued trials than on uncued trials $(M \Delta=.081, S E M \Delta=.01)$. Thus, as expected, there was a reliable voluntary cueing effect that was not significantly modulated by experiment $(p=.160)$.

\section{Spatial congruence}

There was a significant main effect of spatial congruence $(F$ $\left.(2,76)=22.96, p<.001, \eta^{2}=.38\right)$, such that accuracy was significantly reduced when the spatial location of the distracter was incongruent with the target location as compared to when it was congruent $(M \Delta=.014, S E M \Delta=.005, p=.011)$ or absent $(M \Delta=.034, S E M \Delta=.005, p<.001)$. Accuracy was also significantly different between congruent and absent conditions $(M \Delta=.02, S E M \Delta=.005, p<.001)$. Thus, likely due to the greater power of the combined analysis, the reward/ capture effects were reliable both when the distracter appeared in the same and when it appeared in the opposite hemifield as the target. However, the capture effect was significantly greater when the distracter appeared in the opposite than in the same hemifield as the target.

There was no significant interaction of spatial congruence and cue condition $(p=.168)$. However, as expected, there was a significant higher-order interaction between congruence and cue condition with experiment $\left(F(2,76)=3.93, p=.024, \eta^{2}=.09\right)$. This supports our assertion that involuntary reward-driven capture and voluntary attention interact, but that selection-driven capture and voluntary attention do not.

\section{Spatial offset}

There was no main effect of spatial offset $(p=.937)$. The interaction of spatial congruence and spatial offset was significant $\left(F(2,76)=10.19, p<.001, \eta^{2}=.21\right)$, as was the higher order interaction with cue condition $\left(F(2,76)=3.14, p=.049, \eta^{2}=.08\right)$. No interaction with experiment was significant (all $\mathrm{p}>.250$ ). Thus, the voluntary allocation of attention reliably modulates the interaction of spatial congruence with spatial offset, but this effect is not significantly modulated by the presence of a reward-association. 


\section{Discussion}

In the current study we investigated the relationship between current goal-driven factors that voluntarily drive attention and former value-driven factors that involuntarily drive attention. In Experiment 1, voluntary, goal-driven attention and involuntary value-driven attention interacted. The cueing effect was significantly reduced when the reward-associated distracter appeared in the uncued hemifield, indicating that voluntary, goal-driven attention is impacted by involuntary value-driven capture. This observation suggests that when the reward-associated distracter is presented outside the cued hemifield, it is able to widen the attentional window or otherwise redirect attention, which was initially focused by the voluntary cue, disrupting the benefit of the voluntary cue. An additional consequence of this interaction was that the spatial value-driven capture effect was only reliable on cued trials, such that target identification accuracy was reduced when the reward-associated distracter appeared in the opposite hemifield relative the target compared to when it appeared in the same hemifield or was absent. In other words, spatial value-driven capture was only observed when attention could voluntarily be oriented in space. Taken together, the results support the conclusion that although value-driven capture is involuntary, it does not appear to be affected by voluntary attention in the same fashion as involuntary capture by physically salient features, which fail to capture attention when presented at an uncued location (Yantis \& Jonides, 1990; Theeuwes 1991). Thus, the evidence presented in Experiment 1 of this study provides further support that the classic dichotomy of voluntary and involuntary attention is insufficient to describe the factors that drive attention (Awh, Belopolsky, \& Theeuwes, 2012).

This novel finding was accompanied by the replication of both typical value-driven capture and voluntary cueing effects. In Experiment 1 formerly reward-associated features involuntarily captured attention in a subsequent visual search task. When either a high- or low-reward-associated feature was present as a distracter, target accuracy was significantly reduced as compared to when it was absent. The rewardassociated distracter captured attention despite (1) being task-irrelevant, (2) not being physically salient, and (3) no longer being predictive of reward replicating effects previously observed by us (MacLean \& Giesbrecht 2015a, b) and others (Anderson et al., 2011).

In Experiment 1 attention was also voluntarily oriented by a cue indicating the hemifield in which the target would appear, with $100 \%$ validity. Target accuracy was significantly greater on cued trials than on uncued trials, where the cue did not indicate the target hemifield, replicating the classic voluntary cueing effect (Posner, 1980). So, in Experiment 1 there was evidence for both the voluntary allocation of attention and the involuntary allocation of attention by a physically nonsalient, reward-associated feature in the visual search task.
In Experiment 2 we also observed an involuntary capture effect and a voluntary spatial cueing effect, despite the lack of a reward-association. This suggests that selection history alone was sufficient to involuntarily capture attention. However, we did not find that involuntary selection-driven capture interacted with the effects of voluntary attention in the same way that value-driven capture did in Experiment 1. This contrast was further supported by the combined analysis, which found that the interaction of involuntary capture (congruency with the target hemifield) and voluntary attention (the cueing effect) was modulated significantly by the presence of a reward-association (i.e., by experiment). So, the interaction of the spatial effects of involuntary capture and voluntary attention are unique to reward-driven capture.

The results of Munneke et al.'s (2015) study supported their conclusion that the spatial effects of a rewardassociated feature, which are involuntary, and a voluntary spatial cue were independent. Our results support the opposite conclusion. Munneke et al. found that the reward-association did not alter the relationship between the capture by a physically salient distracter and the effects of a voluntary cue (i.e., that the effects were independent regardless of the presence of a reward-association). Our reward-associated feature was not physically salient. It is possible that physical salience modulates the relationship between involuntary value-driven capture and voluntary attention.

It is also possible that the nature of the engagement of voluntary attention differed across studies. Our cues were $100 \%$ valid, whereas Munneke et al. used a cue that was 80 $\%$ valid and was also an overlearned symbol that may have resulted in a kind of involuntary orienting (Ristic \& Kingstone, 2012). It is possible that voluntary attention may exert an effect on involuntary reward-driven capture only when the voluntary cue is entirely predictive, and purely voluntary. Furthermore, the cue Munneke et al. employed, which indicated the exact target location with no uncertainty, may have resulted in a more tightly focused attentional shift. In contrast, the cue in our experiments, which indicated the hemifield in which the target would be presented, but not the exact target location within the hemifield (i.e., less uncertainty, but not none), may have resulted in a more diffuse focus of attention or otherwise larger attended region compared to when a single location is cued. It is possible that the more diffuse attentional window in our experiments allowed for the interaction of value-driven capture with voluntary attention.

There were also differences in temporal contiguity between reward learning and the search task that could account for the discrepant pattern of results. In the current study, the rewardassociation was learned in a training task conducted 1 week prior to the search task. Moreover, in the cued search task, the associations were no longer reinforced, and, thus, the rewardassociations were entirely irrelevant. In contrast, Munneke 
et al. (2015) employed a design in which the rewardassociation was learned concurrently and reinforced throughout the cueing task. The concurrent learning of reward associations and the visual search task may imbue the reward features with task-relevance, albeit spatially nonpredictive. It is also possible that the difference in the dependent variable used to estimate the effects of value-driven capture and cueing - Munneke et al. (2015) used response time, here we used accuracy - also contributed to the contrasting patterns of results. In summary, the results of the current study clearly indicate that irrelevant reward-driven capture can be modulated by voluntary attention, although it may not be under other conditions (i.e., those of Munneke et al., 2015).

Another key finding of Experiment 1 was that while the spatial offset of the distracter from the target alone did not affect target performance, it did interact with the spatial reward-driven capture effect. Specifically, when the target hemifield was cued and the reward-associated feature was presented in the same hemifield (i.e., was congruent), performance degraded as a function of increasing spatial offset between the target and distracter. In contrast, when the distracter with the previously rewarded feature was in the other hemifield (i.e., incongruent), performance improved as a function of spatial offset. This pattern was not observed on uncued trials. The interaction between spatial offset and cued hemifield is consistent with the idea that the rewardassociated distracter helped to further refine attention to the target within the cued, i.e., the voluntarily attended hemifield. However, the reverse is true when the distracter appeared in the uncued hemifield, suggesting that the distracter may be more likely to diffuse attention outside of the cued hemifield, i.e., "widen the attentional window," when the distracter is closer to the target.

Although we did not replicate these effects in Experiment 2, suggesting that they are specific to reward-driven capture, our combined analysis did not find that the presence of a reward-association modulated the effects (i.e., there was no higher-order interaction with experiment). So, based on these data it is unclear whether the spatial offset effects also interact with selection-driven capture as well as reward-driven capture. Although not critical to our interpretation of the results, it is also unclear in the current study the extent to which the observed effects are due to overt versus covert orienting of attention. Participants' gaze was unlimited beyond a strong directive to maintain fixation throughout the experiment. As the cue did not indicate the exact target location it would not have been possible for participants to orient their gaze to any particular target location prior to the stimulus display; however, they may have shifted their gaze off fixation in favor of the cued hemifield. Furthermore, the very brief (220ms) stimulus display would have limited participants' ability, and the relatively small size of the search array (within $\sim 2^{\circ}$ of fixation) their need, to shift their gaze around the stimulus display. In this case, our effects may be due to covert shifts of attention, where the effects observed in previous studies with larger search arrays (e.g., $\sim 5^{\circ}$ of fixation), as in Munneke et al. (2015), may be due to overt shifts.

Although we were able to replicate the overall value-driven capture effect, when examined as a function of congruency with the cued hemifield, we observed that there was no valuedriven capture on uncued trials. This is inconsistent with the wealth of previous research showing value-driven capture effects in paradigms without a spatial cue. However, while the uncued condition in the current study seems equivalent to those previous experiments without spatial cues, it is important to recall that those uncued trials occurred in the context of cued trials. Thus, it appears that when performing a visual search in a task that allows for the voluntary shift of attention prior to target presentation (on some trials), attention is less susceptible to value-driven capture.

The results of the current study not only support the proposal that attention is driven by current goals as well as by factors that, in the past, guided attention toward former goalrelevant information (Awh, Belopolsky \& Theeuwes, 2012), but also that these two factors are not independent. Thus the two sources of goal-driven attention, factors related to current goals, which voluntarily drive attention, and learned factors related to former goals, which can involuntarily drive attention, can and do conflict with each other and this conflict affects the prioritization of attention.

Acknowledgments M.H. MacLean and G.K. Diaz contributed equally to this work. This work was supported by a postdoctoral fellowship from the National Science and Engineering Research Council of Canada to M.H. MacLean, by a research fellowship from the McNair Scholars Program to G.K. Diaz, and by the Institute for Collaborative Biotechnologies through grant W911NF-09-0001 from the U.S. Army Research Office. The content of the information does not necessarily reflect the position or the policy of the Government, and no official endorsement should be inferred.

\section{References}

Anderson, B. A. (2013). A value-driven mechanism of attentional selection. Journal of Vision, 13, 7.

Anderson, B. A. (2014). Value-driven attentional capture is modulated by spatial context. Visual Cognition, (ahead-of-print), 1-15.

Anderson, B. A. (2015). The attention habit: How reward learning shapes attentional selection. Annals of the New York Academy of Sciences.

Anderson, B. A., Laurent, P. A., \& Yantis, S. (2011). Value-driven attentional capture. Proceedings of the National Academy of Sciences, 108(25), 10367-10371.

Awh, E., Belopolsky, A. V., \& Theeuwes, J. (2012). Top-down versus bottom-up attentional control: A failed theoretical dichotomy. Trends in cognitive sciences, 16, 437-443. 
Belopolsky, A. V., Zwaan, L., Theeuwes, J., \& Kramer, A. F. (2007). The size of an attentional window modulates attentional capture by color singletons. Psychonomic bulletin \& review, 14(5), 934-938.

Brainard, D. H. (1997). The Psychophysics Toolbox. Spatial Vision, 10, 433-436.

Bundesen, C. (1990). A theory of visual attention. Psychological review, 97, 523.

Carver, C. S., \& White, T. L. (1994). Behavioral inhibition, behavioral activation, and affective responses to impending reward and punishment: The BIS/BAS scales. Journal of personality and social psychology, 67(2), 319.

Chun, M. M., \& Jiang, Y. (1998). Contextual cueing: Implicit learning and memory of visual context guides spatial attention. Cognitive psychology, 36(1), 28-71.

Corbetta, M., \& Shulman, G. L. (2002). Control of goal-directed and stimulus-driven attention in the brain. Nature reviews neuroscience, $3,201-215$.

Desimone, R., \& Duncan, J. (1995). Neural mechanisms of selective visual attention. Annual review of neuroscience, 18, 193-222.

Eckstein, M. P., Drescher, B. A., \& Shimozaki, S. S. (2006). Attentional cues in real scenes, saccadic targeting, and Bayesian priors. Psychological Science, 17(11), 973-980.

Greenberg, A. S., Esterman, M., Wilson, D., Serences, J. T., \& Yantis, S. (2010). Control of spatial and feature-based attention in frontoparietal cortex. The Journal of Neuroscience, 30(43), 14330-14339.

Hickey, C., Chelazzi, L., \& Theeuwes, J. (2010). Reward changes salience in human vision via the anterior cingulate. Journal of Neuroscience, 30, 11096-11103.

Itti, L., \& Koch, C. (2001). Computational modelling of visual attention. Nature reviews neuroscience, 2, 194-203.

Johnson, D. N., McGrath, A., \& McNeil, C. (2002). Cuing interacts with perceptual load in visual search. Psychological Science, 13(3), 284-287.

Jonides, J., \& Yantis, S. (1988). Uniqueness of abrupt visual onset in capturing attention. Perception \& Psychophysics, 43(4), 346-354.

Kelley, T. A., Serences, J. T., Giesbrecht, B. \& Yantis, S. Cortical mechanisms for shifting and holding visuospatial attention. Cerebral Cortex (2007).

Kleiner M, Brainard D, Pelli D, 2007, "What's new in Psychtoolbox-3?" Perception 36 ECVP Abstract Supplement.

Kyllingsbæk, S., Schneider, W. X., \& Bundesen, C. (2001). Automatic attraction of attention to former targets in visual displays of letters. Perception \& Psychophysics, 63(1), 85-98.

Laurent, P. A., Hall, M. G., Anderson, B. A., \& Yantis, S. (2015). Valuable orientations capture attention. Visual Cognition, 23(1-2), 133-146.

MacLean, M. H., \& Giesbrecht, B. (2015a). Irrelevant reward and selection histories have different influences on task-relevant attentional selection. Attention, Perception, \& Psychophysics, 1-14.
MacLean, M. H., \& Giesbrecht, B. (2015a). Neural evidence reveals the rapid effects of reward history on selective attention. Brain research, 1606, 86-94.

Munneke, J., Hoppenbrouwers, S. S., \& Theeuwes, J. (2015). Reward can modulate attentional capture, independent of top-down set. Attention, Perception, \& Psychophysics, 77(8), 2540-2548.

Pelli, D. G. (1997). The VideoToolbox software for visual psychophysics: Transforming numbers into movies. Spatial Vision, 10, 437-442.

Posner, M. I. (1980). Orienting of attention. Quarterly journal of experimental psychology, 32(1), 3-25.

Remington, R. W., Johnston, J. C., \& Yantis, S. (1992). Involuntary attentional capture by abrupt onsets. Perception \& Psychophysics, 51(3), 279-290.

Ristic, J., \& Kingstone, A. (2012). A new form of human spatial attention: Automated symbolic orienting. Visual Cognition, 20(3), 244-264.

Sali, A. W., Anderson, B. A., \& Yantis, S. (2014). The role of reward prediction in the control of attention. Journal of experimental psychology: human perception and performance, 40(4), 1654.

Serences, J. T., \& Yantis, S. (2007). Spatially selective representations of voluntary and stimulus-driven attentional priority in human occipital, parietal, and frontal cortex. Cerebral Cortex, 17(2), 284-293.

Serences, J. T., Shomstein, S., Leber, A. B., Golay, X., Egeth, H. E., \& Yantis, S. (2005). Coordination of voluntary and stimulus-driven attentional control in human cortex. Psychological Science, 16(2), $114-122$

Sy, J. L., Guerin, S. A., Stegman, A., \& Giesbrecht, B. (2014). Accurate expectancies diminish perceptual distraction during visual search. Frontiers in human neuroscience, 8.

Theeuwes, J. (1991). Exogenous and endogenous control of attention: The effect of visual onsets and offsets. Perception \& psychophysics, 49(1), 83-90.

Theeuwes, J., Kramer, A. F., \& Belopolsky, A. V. (2004). Attentional set interacts with perceptual load in visual search. Psychonomic Bulletin \& Review, 11, 697-702.

Vuilleumier, P. (2015). Affective and motivational control of vision. Current Opinion in Neurology, 28(1), 29-35.

Yantis, S., \& Jonides, J. (1984). Abrupt visual onsets and selective attention: Evidence from visual search. Journal of Experimental Psychology: Human perception and performance, 10, 601-621.

Yantis, S., \& Jonides, J. (1990). Abrupt visual onsets and selective attention: Voluntary versus automatic allocation. Journal of Experimental Psychology: Human perception and performance, 16(1), 121.

Yantis, S., \& Jonides, J. (1996). Attentional capture by abrupt onsets: New perceptual objects or visual masking? Journal of Experimental Psychology: Human Perception and Performance, 22, 1505-1513.

Yantis, S., Schwarzbach, J., Serences, J. T., Carlson, R. L., Steinmetz, M. A., Pekar, J. J., \& Courtney, S. M. (2002). Transient neural activity in human parietal cortex during spatial attention shifts. Nature Neuroscience, 5, 995-1002. 